\title{
Implication of Socio-Demographics on Cognitive-Related Symptoms in Sports Concussion Among Children
}

\author{
Laurens Holmes $\mathrm{Jr}^{1,2^{*}}$, Joshua Tworig ${ }^{2}$, Joseph Casini ${ }^{3}$, Isabel Morgan ${ }^{1,4}$, Kathleen O'Brien ${ }^{5}$, Patricia Oceanic ${ }^{1}$ \\ and Kirk Dabney ${ }^{1,5}$
}

\begin{abstract}
Background: Sports-related concussion remains a public health challenge due to its morbidity and mortality. One of the consequences of concussion is cognitive impairment (Cl) and cognitive-related symptoms (CRS) which determine, to some extent, physical and behavioral functioning of children who sustain concussion. Despite the high prevalence of $\mathrm{Cl}$ and CRS associated with concussion, the risk factors are not fully understood. We aimed to characterize CRS and to examine its relationship with race, ethnicity, age, insurance, and sex in a pediatric population.

Methods: A retrospective cohort (case-only) design was used to assess CRS prevalence and its relationship with race and sex using a pediatric hospital's electronic medical records. A consecutive sample was used with 1429 cases between 2007 and 2014. Study characteristics were examined using chi-square and log binomial regression for hypothesis-specific testing.

Results: Of the 1429 cases, 872 (61.0\%) were boys and 557 (39.0\%) were girls. The racial distribution indicated 1146 (80.2 \%) Whites, 170 (11.9\%) Blacks/African Americans, and 113 (7.9\%) others. The prevalence of CRS was $78.0 \%$. Whereas boys had sustained more concussions, girls were more likely to present with CRS; prevalence risk ratio $=1.07,95 \% \mathrm{Cl} 1.01-1.13, p=0.02$. The crude analysis indicated no racial disparities in CRS prevalence, but the multivariable analysis did, comparing White to Black/African American children; adjusted prevalence risk ratio (aPRR) $=1.77,99 \% \mathrm{Cl} 1.02-3.08, p=0.008$.
\end{abstract}

Conclusions: Racial disparities exist in CRS among children with sports-related concussion, and Black/African American children are more likely, relative to Whites, to suffer CRS. Due to uncertainty in causal inference, we caution the interpretation and application of these data in risk-adapted concussion prevention.

\section{Key Points}

- Cognitive-related symptoms (CRS) are relatively high following mild traumatic brain injury (mTBI).

- While boys sustain more mTBI, CRS in our sample was more prevalent among girls.

- African Americans/Blacks relative to Whites were more predisposed to CRS, an implication of riskadapted intervention in reducing $\mathrm{mTBI}$ and CRS among children.

\footnotetext{
* Correspondence: drholmesjr@gmail.com

${ }^{1}$ Nemours/Alfred I. DuPont Hospital for Children, Office of Health Equity and Inclusion, P.O. Box 269, Wilmington, DE 19803, USA

2University of Delaware, Newark, DE 19716, USA

Full list of author information is available at the end of the article
}

\section{Background}

Approximately 1.6 to 3.8 million traumatic brain injuries (TBIs) occur each year in the USA [1-4]. The majority of these are concussions, a form of mild TBI (mTBI). It is estimated that 300,000 of these concussions are sportsrelated [5]. Since the 1990s, sports concussions have become a major health concern and public health challenge. Evidence of the long-term consequences of concussion has fueled efforts to improve its management. For example, De Beaumont and colleagues recently found that even retired athletes who sustained a concussion as long as 30 years prior to the study had significant cognitive and motor system alterations [6]. This underscores the concern for head injury in sporting activities. Due to the lack of widespread injury surveillance, however, many sports- 
related concussions go unrecognized. Youth athletes are at increased risk due to maximal brain plasticity during development [7]. Furthermore, Gessel et al. found that concussion accounts for more high school athletic injuries than collegiate athletic injuries [8]. In a study conducted by Marar and colleagues, concussion accounted for $13.2 \%$ of all high school athletic injuries [9].

In addition to observed age differences in the incidence of sports-related concussions, a few studies have reported differences by gender and race/ethnicity. While male athletes have a higher concussion incidence overall, concussion incidence is higher among females for gender-comparable sports $[1,8-10]$. Similarly, a large cohort $(n=1425)$ study on sex differences in outcome after mTBI observed significant odds of poorer outcomes following mTBI in girls relative to boys based on the post-concussion symptoms (PCS) score. In this cohort, 3 months after injury, males relative to females had a significant $38 \%$ decreased odds of higher PCS scores, OR, 0.62, $p<0.001$ [11].

Some studies have identified racial/ethnic differences in concussion incidence, awareness, outcome, and mortality. For example, Bloodgood et al. found that Blacks/ African Americans and Hispanics were less likely to be aware of concussions than non-Hispanic Whites [12]. Langlois et al. indicated that death and hospitalization rates for Black/African American children 0-4 years old who sustained TBI were twice those of Whites [5]. Identifying and addressing these differences is essential in designing and implementing intervention efforts to reduce the incidence and complications of concussion among various groups.

One potential consequence of concussion is cognitive impairment, which affects memory, attention, and orientation. Outcomes of concussion in children could be devastating later in life with observed deficits in intellectual functioning and language acquisition. A study by Catroppa et al. assessed adaptive functioning 10 years post-injury in children with early TBI and observed poorer adaptive skills for those with more severe injury and behavioral difficulties [13]. Of the behavioral outcomes reported are difficulties with psychosocial functioning and psychiatric problems later in life $[14,15]$.

Impairment is often measured using one or more standardized test, including the Immediate Post-Concussion Assessment Cognitive Test (ImPACT). Along with sex and racial/ethnic disparities in head injury incidence and outcome, studies have also found evidence of disparities in levels of cognitive impairment after concussion within these groups. For example, results of several studies conducted by Covassin and colleagues revealed that female high school and collegiate athletes exhibited greater impairment in visual memory and more symptoms overall compared with males after concussion $[1,16]$. A similar study conducted by Kontos and colleagues found differences in ImPACT composite scores by race, with Blacks/ African Americans performing worse than Whites after concussion [17]. These outcome differences may be attributable to socioeconomic status and environmental factors, as well as biologic factors inherent within given racial/ethnic groups. A study on the predictors of neuropsychological test scores following mild TBI included race, education, history of learning problems, and days from injury to rehabilitation admission as the significant predictors of cognitive function. Relative to Whites, African Americans which constituted $36.8 \%$ of the total study sample scored lower on all seven neuropsychological tests used for the assessment of cognitive function. The lower scores by African Americans were attributed to unequal access to educational opportunities and cultural differences as well as lack of equivalence in cognitive measures [11].

There remain unidentified factors related to the impact of mTBI and repetitive head trauma among children. These factors include though are not limited to social and cultural differences in predisposition to mTBI. Additionally, very little focus had been on mTBI and the impact of race and ethnicity among other health disparities indicators. Specifically, there are limited consistent data illustrating differences in concussion incidence, prevalence, and severity between boys and girls in sports. A few studies have examined the role of race, ethnicity, and socioeconomic factors in cognitive impairment immediately following concussion in children. Most available data only explore concussions in high school or collegiate athletes, whereas data are limited for children under the age of 13 . The aims of the current study were to (1) characterize sports-related concussion among children, (2) assess the prevalence of cognitive-related symptoms following concussion, and (3) examine the relationship between CRS and race, sex as well as insurance.

\section{Methods}

We conducted a retrospective study to examine sportsrelated concussion in relation to cognitive-related symptoms among children, following institutional review board (IRB) approval. To assess the response or outcome variable, namely cognitive-related symptom, in relation to health disparities indicators, a retrospective cohort design (case-only) was used. This design is effective, in spite of its retrospective nature, in obtaining evidence on the assessed relationship with risk ratio effect size. Using preexisting data from our electronic medical records, we examined all cases with concussion as our cohort. 


\section{Study Population}

The study population comprised children aged 2-19 years, who were diagnosed with sports-related concussion between January 2007 and June 2014. This consecutive sample consisted of all children regardless of race, ethnicity, and sex who met our eligibility criteria. To be included in this sample, participants were (a) diagnosed with concussion or TBI between 2007 and 2014, (b) children 219 years of age, (c) residing in DE Valley, and (d) underwent SCAT evaluation. We utilized consecutive sampling technique, thus including all children with sports-related concussion during the study period, between 2007 and mid-2014. Following the eligibility criteria, there were $n=$ 1429 in our sample, with racial distribution indicating the following: White 1146 (80.2 \%), Black/African American 170 (11.9\%), and other 113 (7.9\%).

\section{Study Variables}

Concussion which is derived from Latin "concutere" refers to violent shaking of the brain, and represents the most common and less severe or mild traumatic brain injury (mTBI). Concussion reflects the disturbance in brain function as a result of direct or indirect force to the head. A direct impact to the brain is not required for concussion to occur, given the nature of this condition to be functional rather than structural injury due to shear stress to brain tissue as an outcome of rotational or angular forces [18]. The impact from concussion may result in bruising, blood vessels damage, and injury to the nerves. The third International Conference on Concussion in sports characterized concussion as a complex pathophysiologic process affecting the brain, induced by traumatic biomechanical forces [18].

\section{Sport-Related Concussion Ascertainment}

Concussion as related above is mild traumatic brain injury and could be characterized as invisible injury due to rapid acceleration or deceleration of brain tissues within the skull. These impacts may result in changes in the shape of the brain, brain stretch as well as brain cell damage. Additionally, the impulsive force may be transmitted beyond the head involving the face, neck, or elsewhere. Participants were involved in organized sports at school (football, baseball, softball, basketball, cheerleading, gymnastics, soccer) and were assessed using the symptom checklist. Girls who played soccer, softball, and basketball while boys who played football presented with majority of mTBI. To assist in the diagnosis of concussion, we used many tools to increase the sensitivity and specificity of the ascertainment. Specifically, we applied the post-concussion symptom scale, and concussion symptom inventory (CSI). Of these two instruments, we based our ascertainment on CSI since this is an empirically derived symptom checklist. Additionally, we used sideline assessment tools mainly Sport Concussion Assessment Tool (SCAT2) since it combines multiple assessment tools as well as physical examination to determine the possibility of concussion as mTBI. Therefore, our final assessment was based on SCAT2, the source for our data on CRS.

Children as patients provided the information during history taking and physical examination clinical encounter. However, for the every young ones, parents and caregivers serve as proxies to the children as respondents. The mean duration between injury and assessment (rehabilitation admission) at our facility was 7.6 days, SD 4.3.

Other variables studied included cognitive-related symptom, race, ethnicity, sex, age, insurance coverage, and length of hospitalization, as well as other variables that were available but not included in the final analysis, namely language, comorbidity, means of hospital arrival, and year of concussion.

\section{Cognitive-Related Symptom (CRS) Ascertainment}

Cognitive-related symptom was available as a string variable and was characterized as memory, orientation, and attention deficit. Specifically, we grouped symptoms and manifestation based on the variables in the medical records that reflected cognitive-related symptoms including a feeling of mental fogginess, problems concentrating, problems remembering, visual disturbance, amnesia, dizziness, a feeling of being slowed down, a feeling of increased emotion, and balance problems. We extracted these variables and used a binary scale to describe cognitive-related symptoms $(0=$ absence, $1=$ presence). Cognitive-related symptom, based on our approach, indicated several attributes for a given case indicative of magnitude or degree of symptoms.

\section{Other Variables Ascertainment (Race, Sex, Insurance, Age} at Injury)

Race and ethnicity were self-reported. We collected data on those who self-identified as White, Black/African American, and some other race. Additional races represented in our data consisted of American Indian/ Alaska Native, Asian, and Hawaiian Native/Other Pacific Islander. Similarly, participants self-identified as nonHispanic/Latino or Hispanic/Latino. Self-reported variables have been utilized and deemed to be reliable with at least an estimated $80 \%$ confidence [19]. Sex was available as male and female and was measured on a nominal scale. As recommended by the American Medical Association, we used boys and girls to represent the sex of our patients [20]. Furthermore, sex was critical to stratify sports-related concussion and was included in prevalence risk ratio test and adjusted risk ratio test. We examined insurance as both a proxy for income and access to available care following concussion. The insurance variable was classified into commercial (private), 
public (Medicaid), and uninsured/self-pay. We assessed the effect of insurance on cognitive-related symptoms using private/commercial insurance as the reference group. Data from our medical records were available for age on a continuous scale. To assess the effect of age on CRS as well as concussion, we utilized age as a continuous variable and transformed the age variable into categories, namely 2-9 years, 10-14 years, and 1519 years. These ages at injury were treated as continuous variables which were later categorized to assess the impact of age on concussion as well as cognitive-related symptom.

\section{Statistical Analysis}

The categorical and nominal variables including the prevalence of CRS were summarized using frequency and percentages, while the continuous scale variable was summarized using median and interquartile range (IQR) depending on the normality assumption. The chi-squared statistic, Pearson, and where necessary Fisher's exact were used to characterize the categorical and nominal variables with concussion and cognitive-related symptoms. To examine the association between race, ethnicity sex, insurance, age, and age group with CRS, univariable and multivariable $\log$ binomial regression models were used. We built a multivariable $\log$ binomial regression model to examine the simultaneous effect of race, sex, insurance, age, and age group on CRS. We used forward loading and backward elimination and included all variables that were significant at 0.25 type I error, potential confounding variables with $10 \%$ difference between the crude and stratified association and those that had a biologic or clinical relevance, such as age and sex. The significance level for the univariable model was 0.05 (95\% confidence interval), while the significance level for the multivariable model was 0.01 ( $99 \%$ confidence interval) as to minimize type I error that may result from multiple comparison in the multivariable log binomial regression model. All tests were two-tailed, and the entire analyses were performed using Stata version 13.0 (Stata Corporation, College Station, Texas) [21].

\section{Results}

Although not shown in the tables, $78.1 \%$ of the children indicated measureable CRS. The prevalence of concussion was greater among boys $(59.3 \%)$ relative to girls $(40.7 \%)$. Table 1 presents the study characteristics of children with sports-related concussion stratified by race. There was a statistically significant sex distribution of children who sustained concussion by race. Among Blacks/African Americans, concussion was relatively higher among boys (71.8 \%) compared to girls (28.2 \%), whereas among Whites, concussion was marginally higher in boys (59.3\%) compared to girls $(40.7 \%), \chi^{2}(2)$ $=9.66, p=0.008$. A statistically significant difference by race was not observed in CRS, $\chi^{2}(2)=0.43, p=0.81$. Insurance coverage (implying access to care) showed a statistically significant variation by race. Whites were more likely to have private/commercial insurance relative to Blacks/African Americans (Whites $90.3 \%$, Blacks/African Americans $63.5 \%), \chi^{2}(4)=105.1, p<0.0001$.

The characterization of the study variables by ethnicity is illustrated in Table 2. Although there was no significant difference in the distribution of sex by ethnicity among children with concussion, non-Hispanic/Latino girls were less likely to have concussion relative to their Hispanic/Latino counterparts. CRS, although statistically insignificant, was more common among Hispanic/Latino children $(81.4 \%)$ relative to non-Hispanic/Latino children $(77.8 \%) \chi^{2}(1)=0.69, p=0.41$. Similar to insurance coverage in the racial distribution, a significant ethnic variation was observed, with non-Hispanic/Latino children more likely to have private insurance compared to Hispanic/Latino children (non-Hispanic/Latino $86.7 \%$, Hispanic/Latino $73.5 \%), \chi^{2}(2)=14.6, p=0.001$.

The crude association between CRS and the independent variables race and sex, as well as other study variables is demonstrated in Table 3. A significant racial variation was not observed with respect to CRS, $p>0.05$. Girls were more likely to have CRS following sports-related concussion, with an observed $7 \%$ increased likelihood compared to boys, risk ratio (RR) $=1.07,95 \% \mathrm{CI} 1.01-1.13, p=0.02$. Although statistically insignificant, compared to children with private insurance, the uninsured children were $6 \%$ more likely to have cognitive impairment following sports-related concussion, $\mathrm{RR}=1.06,95 \%$ CI $0.95-1.18$, $p=0.31$. A significant association was observed between age and CRS, with older children presenting with greater risk. Compared to children 2-9 years, children 10-14 years had $13 \%$ increased risk of sustaining CRS following concussion ( $\mathrm{RR}=1.13,95 \% \mathrm{CI} 1.00-1.28, p=0.06)$ and children $15-19$ years had a $19 \%$ increased risk, $R R=1.19$, $95 \%$ CI 1.05-1.35, $p=0.005$.

The simultaneously adjusted risk model is shown in Table 4. After controlling for insurance, age, sex, and length of hospitalization, Blacks/African Americans had a significant $77 \%$ higher risk of CRS after concussion relative to Whites, adjusted prevalence risk ratio $(\mathrm{aPRR})=$ $1.77,99 \%$ CI, 1.02-3.08.

\section{Discussion}

This study was performed to characterize concussion sustained among children who play sports and were seen in our pediatric institution. We postulated that patients who sustained concussion present with substantial CRS and that differences in outcome may be observed by race, ethnicity, and other health disparities indicators. Using an explanatory multivariable binomial regression (predictive) model, we obtained some relevant findings. 
Table 1 Study characteristics of children with sports-related concussion, stratified by race

\begin{tabular}{|c|c|c|c|c|c|}
\hline \multirow[t]{3}{*}{ Variable } & \multicolumn{3}{|l|}{ Race } & \multirow{3}{*}{$x^{2}(d f)$} & \multirow{3}{*}{$p$} \\
\hline & White & Black/African American & Some Other Race & & \\
\hline & $n(\%)$ & $n(\%)$ & $n(\%)$ & & \\
\hline \multicolumn{4}{|l|}{ Sex } & $9.66(2)$ & 0.008 \\
\hline Male & $680(59.3)$ & $122(71.8)$ & $70(62.0)$ & & \\
\hline Female & $466(40.7)$ & $48(28.2)$ & $43(38.0)$ & & \\
\hline \multicolumn{4}{|c|}{ Cognitive-related Symptoms } & $0.43(2)$ & 0.81 \\
\hline Yes & $899(78.4)$ & $130(76.5)$ & $87(76.3)$ & & \\
\hline No & $247(21.6)$ & $40(23.5)$ & $27(23.7)$ & & \\
\hline \multicolumn{4}{|c|}{ Insurance Coverage } & $105.1(4)$ & $<0.0001$ \\
\hline Private & $1035(90.3)$ & $108(63.5)$ & $83(73.5)$ & & \\
\hline Public & $67(5.9)$ & $43(25.3)$ & $19(16.8)$ & & \\
\hline Uninsured & $44(3.8)$ & $19(11.2)$ & $11(9.7)$ & & \\
\hline \multicolumn{4}{|l|}{ Language } & $0.34(2)$ & 0.84 \\
\hline English & $834(72.8)$ & $123(72.4)$ & $85(75.2)$ & & \\
\hline Spanish & $312(27.2)$ & 47 (27.6) & $28(24.8)$ & & \\
\hline \multicolumn{4}{|l|}{ Year Injured } & $36.2(14)$ & 0.001 \\
\hline 2007 & $6(0.5)$ & $0(0.0)$ & $0(0.0)$ & & \\
\hline 2008 & $33(2.9)$ & $3(1.8)$ & $2(1.8)$ & & \\
\hline 2009 & $134(11.7)$ & 19 (11.2) & $2(1.8)$ & & \\
\hline 2010 & $182(15.9)$ & 38 (22.3) & $11(9.7)$ & & \\
\hline 2011 & 205 (17.9) & $24(14.1)$ & 18 (15.9) & & \\
\hline 2012 & $163(14.2)$ & $26(15.3)$ & $24(21.2)$ & & \\
\hline 2013 & $293(25.6)$ & $50(29.4)$ & $34(30.1)$ & & \\
\hline 2014 & 130 (11.3) & $10(5.9)$ & $22(19.47)$ & & \\
\hline
\end{tabular}

Notes and abbreviations: The type 1 error tolerance (significance level) was set at $5 \%(0.05) . n=$ number, $\%=$ percentage, $d f=$ degree of freedom, Pearson Chi-squared $=x^{2}$

(1) The occurrence of CRS is relatively high among children who play sports and sustained concussion. (2) CRS is associated with sex, with more girls reporting impairment relative to boys. (3) There is a substantial relationship between age and CRS. (4) After controlling for potential confounding, such as insurance, age, sex, and length of hospitalization, Black/African American children were more likely to develop CRS following sportsrelated concussion.

Racial variances in CRS were not observed in our crude (unadjusted) model. This observation may be due to the use of a binary variable in the measurement of CRS. In our data, we observed several indicators of CRS that required the use of ordinal scale to rank this variable. Therefore, the application of ordered category might result in the observation of a significant difference in CRS by race. We built a multivariable model using log binomial regression for possible explanation, adjusting for biologic and known confounding factors for racial association with disease and health conditions. The multivariable explanatory model clearly indicated racial variance in CRS following sports-related concussion. These data consequently support a previous studies in comparable settings. While a prospective case-control study did not observe a significant difference between African and Whites on baseline or post-concussion verbal memory, visual memory, reaction time, and total reported symptoms, however, African Americans were almost 2.5 times as likely to have at least one clinically significant cognitive decline on ImPACT at 7 days postconcussion, as well as scored lower at 7 days postconcussion relative to baseline on processing speed [17]. In another study, relative to Whites, African Americans that constituted $36.8 \%$ of the total study sample scored lower on all seven neuropsychological tests (verbal memory-total and delayed, working memory-WAIS-3 DS and WAIS 3 LNS, perceptual reasoning, visual memory, processing speed) used for the assessment of cognitive function. These racial variances were attributed by authors attributed to unequal access to educational opportunities and cultural differences as well as lack of equivalence in cognitive measures [22]. 
Table 2 Study characteristics of children with sports-related concussion, stratified by ethnicity

\begin{tabular}{|c|c|c|c|c|}
\hline \multirow[t]{3}{*}{ Variable } & \multicolumn{2}{|l|}{ Ethnicity } & \multirow{3}{*}{$x^{2}(d f)$} & \multirow{3}{*}{$p$} \\
\hline & Non-Hispanic/Latino & Hispanic/Latino & & \\
\hline & $n(\%)$ & $n(\%)$ & & \\
\hline \multicolumn{3}{|l|}{ Sex } & $0.80(1)$ & 0.37 \\
\hline Male & $814(61.3)$ & $58(56.9)$ & & \\
\hline Female & $513(38.7)$ & $44(43.1)$ & & \\
\hline \multicolumn{3}{|c|}{ Cognitive-related Symptoms } & $0.69(1)$ & 0.41 \\
\hline Yes & $1033(77.8)$ & $83(81.4)$ & & \\
\hline No & $294(22.2)$ & 19 (18.6) & & \\
\hline \multicolumn{3}{|c|}{ Insurance Coverage } & $14.6(2)$ & 0.001 \\
\hline Private & $1151(86.7)$ & $75(73.5)$ & & \\
\hline Public & $110(8.3)$ & 19 (18.6) & & \\
\hline Uninsured & $66(5.0)$ & $8(7.8)$ & & \\
\hline \multicolumn{3}{|l|}{ Year Injured } & $21.3(7)$ & 0.003 \\
\hline 2007 & $6(0.5)$ & $0(0.0)$ & & \\
\hline 2008 & $37(2.8)$ & $1(1.0)$ & & \\
\hline 2009 & $149(11.2)$ & $6(5.9)$ & & \\
\hline 2010 & $223(16.8)$ & $8(7.8)$ & & \\
\hline 2011 & $233(17.6)$ & $14(13.7)$ & & \\
\hline 2012 & $197(14.8)$ & $16(15.7)$ & & \\
\hline 2013 & $341(25.7)$ & $36(35.3)$ & & \\
\hline 2014 & $141(10.6)$ & $21(20.6)$ & & \\
\hline
\end{tabular}

Notes and abbreviations: The type 1 error tolerance (significance level) was set at $5 \%(0.05) . n=$ number, $\%=$ percentage, $d f=$ degree of freedom

Pearson Chi-squared $=x^{2}$

The observed racial variation in CRS following concussion may be due to the delay in diagnosing and treating concussion, which correlates with poor prognosis. Our finding is supported by a few studies in prior research [17] that examined post-concussion ImPact composite score. The differences in these scores with Blacks/ AA performing poorer relative to Whites may be attributed to several factors including baseline variance, stop socioeconomic status that may impact on timely access to care. While concussion has been shown in literature to vary in prevalence and incidence by race, its sequelae has not been fully described using health disparities indicators. Our study therefore represents a preliminary finding in this vein.

Whereas sports-related concussion has been previously described with respect to sex, however, this study described the prevalence of CRS following mTBI by sex. We have demonstrated that, following concussion, girls have increased risk of sustaining cognitive impairment. While this finding is interesting, we are unable to provide any biologic or psychosocial explanation to justify our observation. However, it is plausible to suggest that it may require longer duration for girls to be seen if
Table 3 Univariable log binomial regression model of the effect of race and other factors on cognitive-related symptoms

\begin{tabular}{|c|c|c|c|}
\hline Variable & $\begin{array}{l}\text { Prevalence } \\
\text { Risk Ratio (RR) }\end{array}$ & $95 \% \mathrm{Cl}$ & $p$ \\
\hline \multicolumn{4}{|l|}{ Race } \\
\hline White & 1.0 & Referent & Referent \\
\hline Black/African American & 0.97 & $0.89-1.07$ & 0.57 \\
\hline Some Other Race & 0.98 & $0.88-1.09$ & 0.73 \\
\hline \multicolumn{4}{|l|}{ Sex } \\
\hline Male & 1.0 & Referent & Referent \\
\hline Female & 1.07 & $1.01-1.13$ & 0.02 \\
\hline \multicolumn{4}{|l|}{ Insurance Coverage } \\
\hline Private & 1.0 & Referent & Referent \\
\hline Public & 1.0 & $0.90-1.10$ & 0.92 \\
\hline Uninsured & 1.06 & $0.95-1.18$ & 0.31 \\
\hline \multicolumn{4}{|l|}{ Age Group } \\
\hline $2-9$ & 1.0 & Referent & Referent \\
\hline $10-14$ & 1.13 & $1.00-1.28$ & 0.06 \\
\hline $15-19$ & 1.19 & $1.05-1.35$ & 0.005 \\
\hline \multicolumn{4}{|l|}{ Language } \\
\hline English & 1.0 & Referent & Referent \\
\hline Spanish & 0.96 & $0.90-1.03$ & 0.25 \\
\hline
\end{tabular}

Notes and abbreviations: The type 1 error tolerance (significance level) was set at $5 \%(0.05) . n=$ number, $\%=$ percentage

Table 4 Multivariable log binomial regression model for the effect of race/ethnicity on cognitive-related symptoms

\begin{tabular}{llll}
\hline Variable & $\begin{array}{l}\text { Adjusted } \\
\text { Risk Ratio }\end{array}$ & $99 \% \mathrm{Cl}$ & $p$ \\
\hline Race & 1.0 & Referent & Referent \\
$\quad$ White & 1.77 & $1.02-3.08$ & 0.008 \\
$\quad$ Black/African American & 0.7 & $0.10-4.93$ & 0.64 \\
$\quad$ Some other race & & & \\
Sex & 1.0 & Referent & Referent \\
Male & 0.79 & $0.39-2.04$ & 0.53 \\
$\quad$ Female & & & \\
Insurance coverage & 1.0 & Referent & Referent \\
Private & 0.72 & $0.30-1.71$ & 0.33 \\
Public & & & \\
Age group & 1.0 & Referent & Referent \\
2-9 & 3.62 & $0.66-19.86$ & 0.052 \\
10-14 & 5.61 & $0.48-65.26$ & 0.07 \\
15-19 & 1.01 & $0.99-1.02$ & 0.19 \\
\hline Length of stay (days) & &
\end{tabular}

Notes and abbreviations: The type 1 error tolerance (significance level) was set at $1 \%(0.01) \cdot n=$ number, $\%=$ percentage, $d f=$ degree of freedom, Pearson Chi-squared $=x^{2}$ 
sports activities are not very well developed in their institutions, implying lack of resources and timely intervention. Despite these difficulties in arriving at a specific explanation, studies have indicated the magnitude and severity of concussion among males relative to females [23]. Differences are suggested to be attributable to differences in head mass and acceleration, as well as other biologic determinants of altered mentation and visual acuity [24]. Furthermore, the increased prevalence in CRS among girls may be attributable to their increasing willingness to disclose their symptoms compared to boys. At this particular competitive level, boys may not like to disclose their symptoms, since doing so may limit their participation in sports [16]. Additionally, a study on sex differences in outcome after mTBI observed significant odds of poorer outcomes following mTBI in girls relative to boys based on the post-concussion symptoms (PCS) score [11]. In effect, we caution the interpretation of increased prevalence in CRS among girls without a careful assessment of other factors related to pain perception and expression.

Insurance has been reported in previous literature to facilitate access and utilization of care. Our studies showed that Blacks/African Americans were less likely to have private insurance compared to their White counterparts, aligning with previous findings illustrating poor outcomes for children with public insurance as well as those who are uninsured [25]. The observed association between insurance coverage and CRS in our sample may be due to decreased access to care as well as decreased care utilization by Blacks/African Americans.

The age at injury was shown in our data to significantly influence CRS. Indeed, we observed a significant association between age and CRS, with older children presenting more with CRS following concussion. The observed association may be due to participation in high-energy contact sports by older children. Willer et al. observed that older children were more likely to sustain head injuries and symptoms of concussion from sports activities, while primary cause of injury for young children was fall [26]. These sports are available to older children and are conducive to an increase in the degree of trauma, which in turn compromises cognitive functions, such as attention, memory, and orientation. It is also worthwhile to consider the changing plasticity of the brain as the child grows, implying the greater ability of the young brain to adjust to change during injury compared to older children with increased vulnerability to mTBI. McKinlay and Anderson examined age at injury as one of the issues associated with preschool child traumatic brain injury. Firstly, this study acknowledged the communication difficulties that may complicate deficits assessment in younger children. Secondly, that the absence of deficit should be cautiously interpreted since the problem or deficit may become apparent over time as the age-dependent skills fail to emerge [27, 28]. Subsequently, our data the association between age at injury and $\mathrm{mTBI}$ supports the observation that neuroplasticity should not be interpreted as the capability of the young child brain to return to pre-injury function [27].

This study is not without limitations. (1) This is a retrospective design that may be influenced by information and selection biases, as well as the lack of temporality, implying cause and effect association. (2) The use of preexisting data from our electronic medical records precluded data collection of known variables that could provide further explanation regarding the relationship between cognitive impairment and race. As a result of this limitation, it is possible to suspect unmeasured confounding to influence the result in this study. (3) In addition, like in most epidemiologic studies, residual confounding may influence the findings in this sample, since no matter how sophisticated a model used for adjusting for confounding, residual confounding persists [29].

\section{Conclusions}

In summary, there are racial, age, and sex disparities with respect to CRS following sports-related concussion in children. However, due to the retrospective nature of our design, caution should be exercised in the interpretation and application of these findings to public health interventions focused on minimizing CRS following sports-related concussions.

\section{Acknowledgements \\ Thanks are due to the Nemours Data Warehouse for the availability of the data used in this study. The responsibility for the analysis of these data remained that of the investigators and not that of Nemours data warehouse. We thank Alex Lahurd, Grace Kalmus, Kristen Juhrden, and Nick Jennelly for the critical reading of the manuscript.}

\section{Funding}

No financial support was received for the conduct of this study or preparation of this manuscript.

\section{Authors' Contributions}

LH conceptualized and designed the study, performed the statistical analysis, interpreted the results, prepared the manuscript, and reviewed and approved the final draft. JT extracted the data from the Electronic Medical Records (EMR), processed the data, assisted in the descriptive analysis and manuscript preparation, and approved the final draft. JC assisted with the data processing, results tabulation, manuscript preparation and approved the final draft. IM assisted with the analysis, results interpretation, manuscript preparation, review and final draft approval. KOB gathered the clinical data for the EMR, assisted in the study conceptualization, and reviewed and approved the final draft. PO assisted in the conduct and study protocol compliance, manuscript review, and the approval of the final draft. KD conceptualized the study, facilitated the preparation of the manuscript, provided critical reading, and approved the final draft.

\section{Competing Interests}

Laurens Holmes Jr., Joshua Tworig, Joseph Casini, Isabel Morgan, Kathleen O'Brien, Patricia Oceanic, and Kirk Dabney declare that they have no conflict of interest. 


\section{Author details}

${ }^{1}$ Nemours/Alfred I. DuPont Hospital for Children, Office of Health Equity and Inclusion, P.O. Box 269, Wilmington, DE 19803, USA. ${ }^{2}$ University of Delaware, Newark, DE 19716, USA. '3Villanova University, Villanova, PA 19085, USA.

${ }^{4} J o h n s$ Hopkins Bloomberg School of Public Health, Baltimore, MD 21205, USA. ${ }^{5}$ Orthopedic Department, Nemours/A. I. duPont Hospital for Children, Wilmington, DE 19803, USA.

Received: 9 February 2016 Accepted: 3 August 2016

Published online: 14 September 2016

\section{References}

1. Covassin T, Swanik CB, Sachs ML. Sex differences and the incidence of concussions among collegiate athletes. J Athl Train. 2003;38(3):238-44.

2. Covassin T, Elbin RJ. The female athlete: the role of gender in the assessment and management of sport-related concussion. Clin Sports Med. 2011;30(1): 125-31.

3. Kerr ZY, Evenson KR, Rosamond WD, Mihalik JP, Guskiewicz KM, Marshall SW. Association between concussion and mental health in former collegiate athletes. Inj Epidemiol. 2014;1:28.

4. Stewart TC, Gilliland J, Fraser DD. An epidemiologic profile of pediatric concussions: identifying urban and rural differences. I Trauma Acute Care Surg. 2014;76(3):736-42.

5. Langlois JA, Rutland-Brown W, Thomas KE. The incidence of traumatic brain injury among children in the United States: difference by race. J Head Trauma Rehabil. 2005:20(1):229-38.

6. De Beaumont L, Tremblay S, Henry LC, Poirier J, Lassonde M, Theoret H. Motor system alterations in retired former athletes: the role of aging and concussion history. BMC Neurol. 2013;13:109.

7. Choe MC, Babikian T, DiFiori J, Hovda DA, Giza CC. A pediatric perspective on concussion pathophysiology. Curr Opin Pediatr. 2012;24(6):689-95.

8. Gessel LM, Fields SK, Collins CL, Dick RW, Comstock RD. Concussions among United States high school and collegiate athletes. J Athl Train. 2007;42(4): 495-503.

9. Marar M, Mcllvain NM, Fields SK, Comstock RD. Epidemiology of concussions among United States high school athletes in 20 sports. Am J Sports Med. 2012; 40(4):747-55.

10. Lincoln AE, Caswell SV, Almquist JL, Dunn RE, Norris JB, Hinton RY. Trends in concussion incidence in high school sports: a prospective 11-year study. Am J Sports Med. 2011;39(5):958-63.

11. Bazarian JJ, Blyth B, McDermott MP, et al. Sex differences in outcome after mild traumatic brain injury. J Neurotrauma. 2010;27:527-39.

12. Bloodgood B, Inokuchi D, Shawver W, Olson K, Hoffman R, Cohen E, Sarmiento K, Muthuswamy K. Exploration of awareness, knowledge, and perceptions of traumatic brain injury among American youth athletes and their parents. J Adolesc Health. 2013;53(1):34-9.

13. Catroppa C, Godfery G, Rosenfeld J, et al. Functional Recovery 10 years following pediatric traumatic brain injury: outcomes and predictors. J Neurotrauma. 2012;29:2539-47.

14. McKinlay A. Injury in preschool-age children. In:Mild Traumatic Brain injury. In: Kirkwood M, Yates K, editors. Children and Adolescents. New York: Guildford Press; 2012.

15. McKinlay A, Dalrymple-Alford J, Horwood L, Fergusson D. Long-term psychosocial outcomes after mild traumatic brain injury in early childhood. J Neurol Neurosurg Psychiatry. 2002;73:281-8.

16. Covassin T, Elbin RJ, Bleecker A, Lipchik A, Kontos AP. Are there differences in neurocognitive function and symptoms between male and female soccer players after concussions? Am J Sports Med. 2013;41(12):2890-5.

17. Kontos AP, Elbin 3rd RJ, Covassin T, Larson E. Exploring differences in computerized neurocognitive concussion testing between African American and White athletes. Arch Clin Neuropsychol. 2010;25(8):734-44.

18. McCrory P, Meeuwisse W, Johnston K, et al. Consensus statement on concussion in sport: the 3rd international conference on concussion in sports held in Zurich, November 2008. J Athl Train. 2009;44(4):434-48.

19. Zuckerman SL, Apple RP, Odom MJ, Lee YM, Solomon GS, Sills AK. Effect of sex on symptoms and return to baseline in sport-related concussion. J Neurosurg Pediatr. 2014;13(1):72-81.

20. "Opinion 8.095 - Reporting clinical test results: general guidelines." In: American Medical Association. AMA, 1998. http://www.ama-assn.org/ama/ pub/physician-resources/medical-ethics/code-medical-ethics/opinion8095. page. Accessed 6 July 2015.
21. STATA Data Analysis and Statistical Software. Vers. 13.1. College station. TX: StataCorp LP: Computer software; 2013.

22. Macciocchi SN, Seel RT, Thompson N. The impact of mild traumatic brain injury on cognitive functioning following co-occuring spinal cord injury. Arch Clin Neuropsychol. 2013;28:684-91.

23. Frommer LJ, Gurka KK, Cross KM, Ingersoll CD, Comstock RD, Saliba SA. Sex differences in concussion symptoms of high school athletes. J Athl Train. 2011:46(1):76-84.

24. Tierney RT, Sitler MR, Swanik CB, Swanik KA, Higgins M, Torg J. Gender differences in head-neck segment dynamic stabilization during head acceleration. Med Sci Sports Exerc. 2005;37(2):272-9.

25. Haider AH, Chang DC, Efron DT, Haut ER, Crandall M, Cornwell 3rd EE. Race and insurance status as risk factors for trauma mortality. Arch Surg. 2008; 143(10):945-9.

26. Willer B, Dumas J, Hutson A, Leddy J. A population based investigation of head injuries and symptoms of concussion of children and adolescents in schools. Injury Prevention. 2004;10:144-8.

27. McKinlay A, Anderson V. Issues associated with pre-school child traumatic brain injury. Available at; http://www.internationalbrain.org/issuesassociated-with-preschool-child-traumatic-brain-injury/retrived05/05/2016.

28. McKinlay A, Grace R, Horwood L, et al. Prevalence of traumatic brain injury among children, adolescents and young adults: prospective evidence from a birth cohort. Brain Inj. 2008;22:175-81.

29. Holmes Jr L, Chan W, Jiang Z, Du XL. Effectiveness of androgen deprivation therapy in prolonging survival of older men with locoregional prostate cancer. J Prostate Cancer Prostatic Dis. 2007;10(4):388-95.

\section{Submit your manuscript to a SpringerOpen ${ }^{\circ}$ journal and benefit from:}

- Convenient online submission

- Rigorous peer review

- Immediate publication on acceptance

- Open access: articles freely available online

- High visibility within the field

- Retaining the copyright to your article

Submit your next manuscript at $\gg$ springeropen.com 